\title{
A simple and Inexpensive click-free audio switch
}

ARNOLD M. SMALL, JR., UNIVERSITY OF IOWA, lowa City, Jowa 52240

This paper describes a click-free audio switch that functions by controlling the rate of onset and decay of a signal. The principle component of the switch is a device whose resistance is a function of the light intensity falling upon it. Circuit diagrams and suggested applications are provided.

Acoustic signals are among the most commonly used stimuli in psychological experimentation. Their use ranges from human psychoacoustic investigations to conditioning studies with rats and other laboratory animals. One of the reasons for this wide use is ease of signal generation. If the stimulus is initially generated in electrical form, then waveform, frequency, and intensity may all be manipulated electrically, with the final operation being the conversion of the electric into acoustic energy by a loudspeaker or earphone. The same ease of manipulation does not exist, at least to the same degree, for most other stimuli.

While the previously mentioned advantages are real, there are a number of precautions a careful $E$ should consider. One deals with the effect of any change in the signal. The most common is an intensity change, specifically the transition from "no signal" to the presence of a signal and back again to the "no signal" condition. If the signal is a sine wave then any change, including the signal onset and termination, introduces acoustic energy at frequencies other than the nominal frequency of the sine wave. Thus, if the sine wave had a nominal frequency of $1000 \mathrm{~Hz}$ at the moment of signal onset and offset, acoustic energy would be spread to frequencies above and below $1000 \mathrm{~Hz}$. One of the perceptual consequences of this energy spread, in humans at least, is the production of a click at the instant of the intensity transition. This effect is not a result of instrument (switch) failure, but a natural and inevitable consequence of intensity change. The degree to which this effect occurs depends upon the rate of the transition, being most marked for abrupt transitions. Perhaps the easiest way to turn a stimulus on and off is with a mechanical switch or a relay. Unfortunately such a switching operation produces an almost instantaneous change in intensity with the widest possible energy spread.

Ordinarily, if a pure tone is presented it is assumed that the $S$ is responding to the tone itself. However, if the stimulus is not actually constant as a function of time (i.e., energy spread at onset and offset), it is possible that the $S$ will respond to some selected aspect of the stimulus. The $\mathrm{E}$ has no simple way of knowing what aspect is serving as the effective stimulus. For example, if a stimulus frequency to which an $S$ is relatively insensitive is used and an abrupt onset and decay is employed, energy will spread to other frequencies including those to which the $S$ is quite sensitive. As a result, the $S$ may respond to that energy contained in the frequency region to which he is sensitive rather than to the nominal stimulus located in an insensitive region. Such considerations become especially important if the $E$ 's interest is in measuring behavior as a function of some dimension of the stimulus, e.g., in studies of discrimination, stimulus generalization, or intensity thresholds, to name just a few.

Energy spread associated with stimulus onset and offset cannot be eliminated, but it can be dramatically minimized. This can be accomplished simply by turning the stimulus on and off gradually rather than abruptly. Although the answer is conceptually straightforward, it cannot be effected by a mechanical contact closure and generally requires electronic switching. Equipment to accomplish this has been available commercially for 20 years, but it is rather complex and expensive. The purpose of this report is to describe a simple, inexpensive switching device that controls the onset and decay times of the stimulus so as to minimize energy spread and its associated problems.

\section{INSTRUMENTATION}

Semiconductor photo-diodes, although differing in details of construction, have a common feature-namely, their electrical resistance is a function of the light to which they are exposed. These devices possess several characteristics that suggest their use in audio-switching circuits: (1) their resistance follows changes in light intensity without appreciable lag in time, (2) their self-generated noise is extremely low, and (3) they are very rugged and reliable. Further, they are available in a configuration containing a built-in light source as well as a unit that contains only the photo-resistive element.

Figure 1 shows one of the simplest possible audio-switching circuits. It operates in the following manner. At a particular moment the mechanical switch, $\mathrm{S}$, is closed allowing current from the voltage source, $E$, to flow through the light source, $L$. Assume for the moment that $L$ is an incandescent source, in which case the light output will lag the applied voltage. That is, it will take some time for the source filament to heat to a steady-state value and, thus, the light output grows slowly as the filament heats. In its "off state," that is, with no light applied, the photo-resistive device, P-R, has a high resistance of perhaps $10^{7} \mathrm{ohms}$. Thus, the current flow through the loudspeaker is extremely small (or in voltage terms, the voltage drop across the P-R device is extremely large compared to that across the loudspeaker). Under these conditions, then, no sound is generated by the loudspeaker.

In its "on state" the P-R device has a low resistance of perhaps 100 ohms. Consequently, a sizable current flows through the loudspeaker and sound is generated. The intensity of the sound is dependent on the magnitude of the current flow through the loudspeaker; this in turn depends upon the ohmic value of the P-R device, which depends upon the light intensity falling upon it. Thus, if the light intensity builds gradually with time (because of the thermal lag of the incandescent filament) so also will the sound intensity. A similar effect occurs as the switch, $\mathrm{S}$, is opened. The filaments take an appreciable time to cool; therefore, the light output decreases gradually and consequently the sound is turned off gradually.

The light source, L, in Fig. 1 may be energized by either dc or ac current. If ac is used, however, it is necessary that the

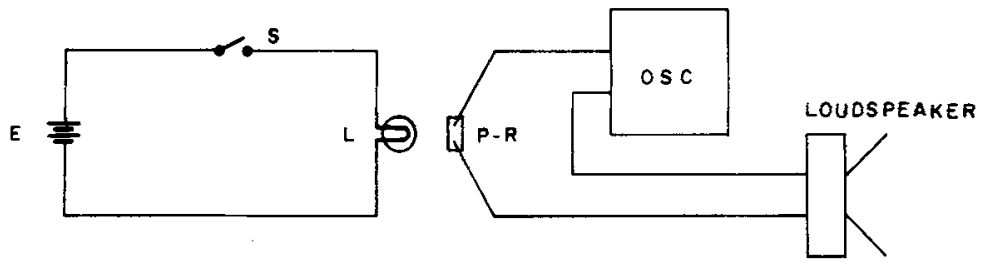

Fig. 1. Simple audio switching circuit using a photo-resistive element as the switching device. 


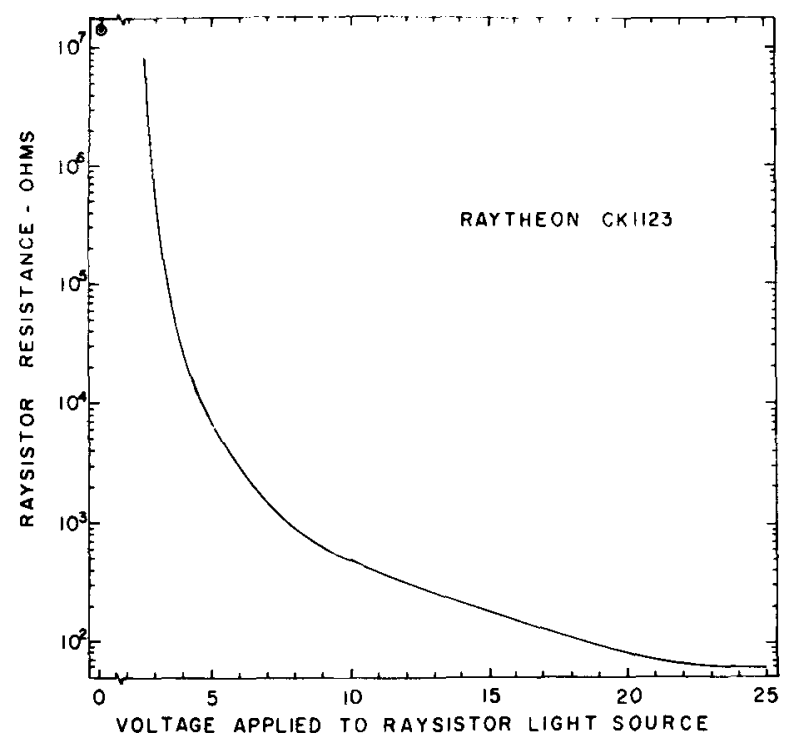

Fig. 2. Resistance value as a function of input voltage to the incandescent light source for the Ray theon CK 1123 "Raysistor." Raysistor is a tradename for a light source and photo-resistive element assembled in a light-tight case.

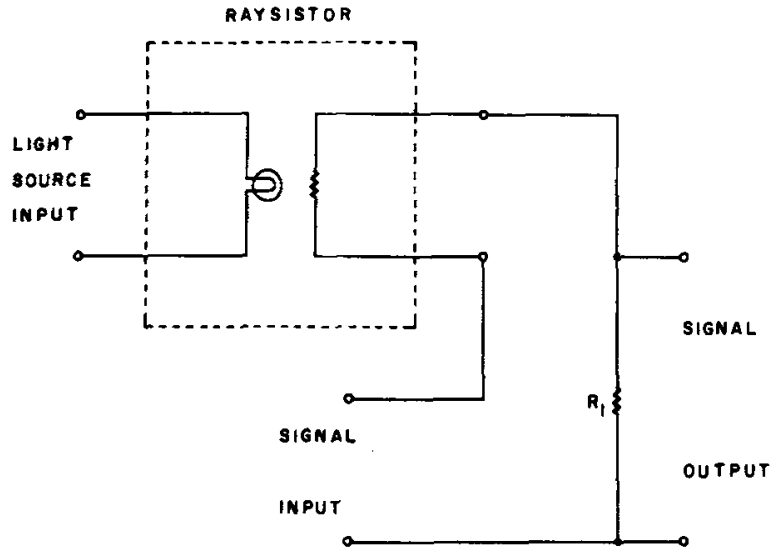

Fig. 3. Raysistor switching circuit showing audio signal input and output. $R_{1}$ is chosen so that when the switch is "on" (resistance of P-R element is low), the design input and output impedance are maintained.

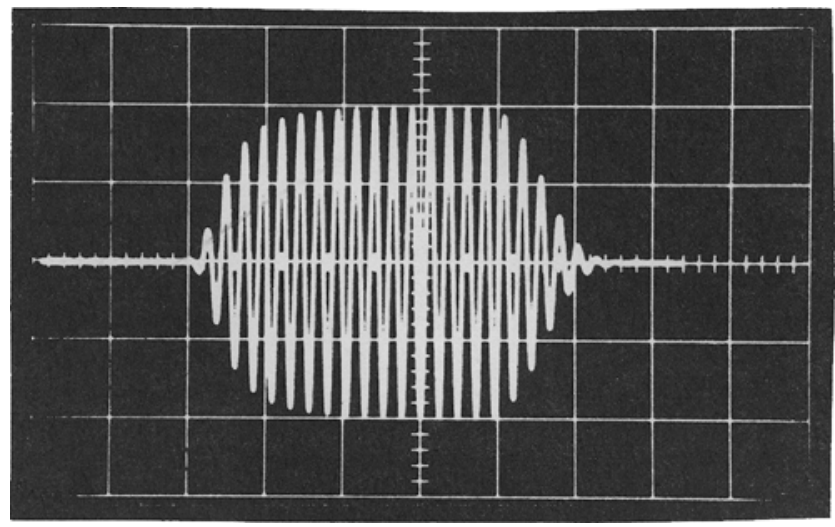

Fig. 5. A photograph of a cathode ray oscilloscope display of a stimulus generated by the devices shown in Figs. 3 and 4. Each major horizontal scale division is equal to $.1 \mathrm{sec}$ and the frequency of the sine wave is $40 \mathrm{~Hz}$.

frequency be high enough so that no variation in light output occurs as a function of time (ripple). If an incandescent source is used, the $60-\mathrm{Hz}$ power-line frequency is satisfactorily high.

The audio switch in the circuit shown in Fig. 1 depends upon characteristics of the light source to provide the gradual onset and decay of the acoustical signal. Thus, for a given light source, the onset and decay characteristics are fixed. However, some variation in these characteristics may be achieved by using different types of incandescent sources (the thermal time constants are influenced by the diameter of the filament wire, for example). Typical "on" switching time is about $30 \mathrm{msec}$ while "off" switching time is about $150 \mathrm{msec}$. These values are quite satisfactory in producing minimal energy spread at stimulus onset and offset (American Standards Association, 1951; Wright, 1960). If for some reason more rapid switching times are required, an ionized gas light source, such as a neon bulb, may be used.

At least one manufacturer (Raytheon) provides the light source and P-R element in a single light-tight package. Such units are available in a wide variety of resistance values and switching times and vastly simplifies construction of devices such as shown in Fig. 1. Figure 2 shows the resistance of the P-R element as a function of the voltage applied to the light source in a typical unit. It should be noted that the on-off ratio-that is, the ratio of the current flowing through the load (a loudspeaker, perhaps) when the switch is "on" compared to the current flow when the

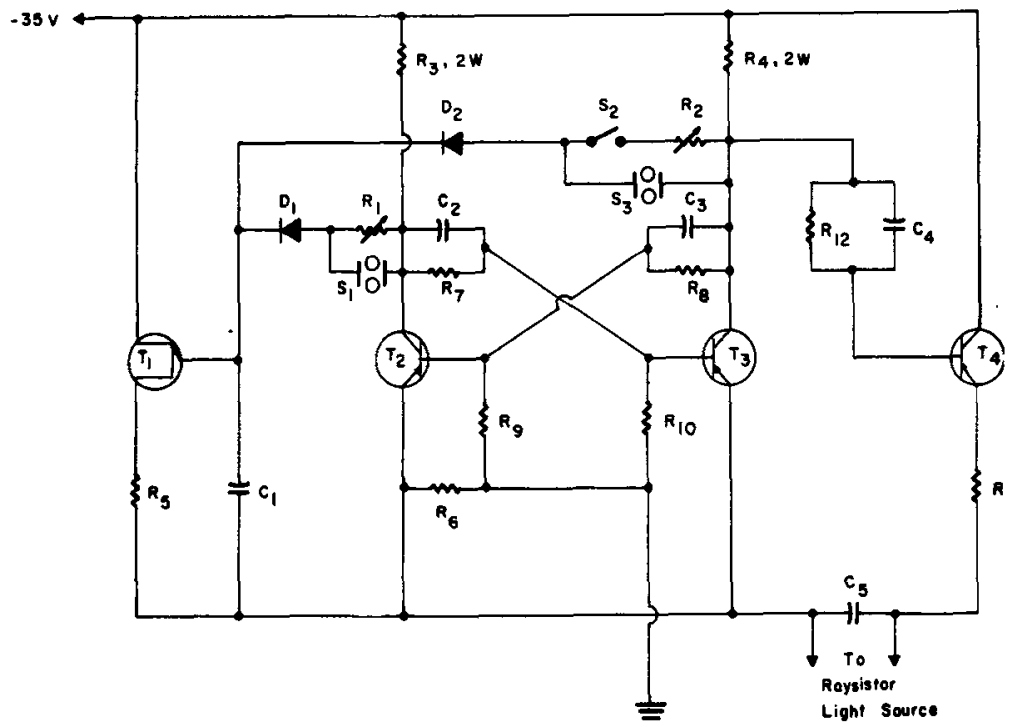

Fig. 4. Light source driver. All resistance values are given in ohms and each unit is $1 / 2 W$ unless otherwise noted: $R_{1}, R_{2}=250,000 ; R_{3}, R_{4}, R_{10}, R_{11}=470$; $R_{5}=330 ; R_{6}=47 ; R_{8}, R_{9}, R_{10}, R_{13}=10,000$ $R_{12}, R_{14}=47,000$. The value of each capacitor is given in microfarads: $C_{1}=25 ; C_{2}, C_{3}=.01 ; C_{4}=.1$; $C_{5}=150 . D_{1}$ and $D_{2}$ are diodes with a reverse a resistance greater than 1000 megohms while $T_{1}$ is a $2 N_{1671}, T_{4}$ is a $2 N 1305$, and $T_{2}, T_{3}$ are $2 N 525 . S_{1}$ and $S_{3}$ are momentary SPST pushbutton switches which serve reset and trigger functions, respectively, when operating in the single-shot mode. When $S_{2}$ is open, operation is single-shot; if closed, operation is free-running. 
switch is "off"-is directly dependent upon the resistance of the P.R element in the "on" and "off" states. To maximize this ratio, the extremes of applied voltage should be used, subject to the limitation of damage to the light source.

Figure 3 shows a schematic drawing of an audio switch employing a P-R element in combination with a light source (a Raytheon CK1123 "Raysistor") used in our laboratory. Usually in psychophysical studies in which it is necessary to control the intensity of audio stimuli, an attenuator is inserted in the circuit immediately following the switching device. In such cases it is desirable to match impedances between adjacent devices for the "switch on" condition. This is accomplished by choosing the proper value for $R_{1}$ (if the "on" resistance of the Raysistor is $60 \mathrm{ohms}$ and the source and load resistance is $500 \mathrm{ohms}$, then $R_{t}$ should equal 3.6 kilohms). The device in Fig. 3 has an on-off ratio in excess of $85 \mathrm{~dB}$ and insertion loss of $3 \mathrm{~dB}$ when working with a 500-ohm source and load resistance. If in Fig. 1 the resistance of the P-R device is represented as $R_{R}$ and that of the loudspeaker as $R_{L}$, then the on-off ratio is given by

$$
\frac{R_{R} \text { "on" }+R_{L}}{R_{R} \text { "off" }+R_{L}}
$$

If $R_{L}=10, R_{R}$ "on" $=60$, and $R_{R}$ "off" $=10^{7}$ ohms, the on-off ratio is $103 \mathrm{~dB}$.

Figure 4 shows a schematic drawing of a control device to provide the input to the Raysistor light source. It consists of a unijunction transistor operating as an astable multivibrator driving a bistable multivibrator that in turn supplies the current to the Raysistor light source through an isolating emitter follower. The circuit provides independent control of on- and off-time when cycling repetitively and of on-time when operating under "one-shot" conditions. Circuit Elements $C_{4}, C_{5}$, and $R_{12}$ provide the necessary shaping of the current onset and offset to provide equal rise and decay times for the audio signal. Figure 5 illustrates the form of a typical sine wave burst. The cost of the Raysistor used here was less than $\$ 4.00$; the cost of the parts contained in the light driver shown in Fig. 4 was under $\$ 15.00$.

\section{DISCUSSION}

In summary, the minimization of the click associated with the onset and termination of an auditory stimulus is important for almost any experiment employing such a stimulus. This click is basically a function of the spectral energy spread associated with abrupt changes in the signal. By controlling the rate of change of signal amplitude at onset and offset, the energy spread is minimized and the perception of the click eliminated. A simple device for accomplishing this consists of a light source and a resistive element whose resistance is a function of the light intensity falling upon it. The variable resistance functions as a graded switching device whose temporal characteristics depend largely upon those of the light source (and its driving current). The advantages of such a switch over commercially available electronic switches are: low cost, low noise level, absence of harmonic distortion, high on-off ratio, high reliability, and uniform frequency response from dc to the megahertz range.

\section{REFERENCES}

WRIGHT, H. N. Audibility of switching transients. Joumal of the Acoustical Society of America, 1960, 32, 138.

AMERICAN STANDARDS ASSOCIATION. American standards specification for audiometers for general diagnostic purposes Z24.5-1951. ASA, New York, March 21, 1951.

\section{An automated multi-channel programming, data collection, and printout system for avoidance and stress conditioning of monkeys}

\section{RONALD I. BARENSTEN and JOAN S. LOCKARD, UNIVERSITY OF WASHINGTON, Seattle, Washington 98105}

A multichannel programming, data collection, and printout system is described for the investigation of avoidance and stress conditioning of monkeys, individually or yoked.

An automated system was developed for simultaneous avoidance or stress conditioning of several yoked pairs or of individual monkeys. During testing each monkey is housed in a primate restraining chair instrumented with a joy-stick lever for avoidance electric shock and a stimulus light panel indicating conditioning treatments. Three treatments may be programmed singly or in all possible combinations: (a) a relatively intense body shock ( $1 / 2-20 \mathrm{~mA})$ for $1 / 2-2 \mathrm{sec}$ duration, delivered periodically (every $5-100 \mathrm{sec}$ ) unless the $S$ resets the interval timer by a lever pull; (b) a relatively low-intensity shock $(1 / 10-5 \mathrm{~mA})$ to the body and hand, for the duration of a lever pull, each time the animal pulls the lever; and (c) an occasional aperiodic unavoidable body shock, recurring every $8 \mathrm{~min}$ on the average, of relatively high intensity $(1 / 2-20 \mathrm{~mA})$ for $1 / 2-2 \mathrm{sec}$ duration. For the initial training, the first treatment may be controlled manually to inhibit programmed shock or deliver unprogrammed shock.

For Treatments (a) and (c) the shock is delivered to the body of the $S$ between a neck-chain and metal neck contact-plate and either the buttocks or feet so that the $S$ cannot avoid the shock by any positioning effort. For Treatment (b) the lever, when pulled, is also in the shock circuit, i.e., the $S$ receives the shock at the hand as well as the body, or only the body depending upon the position of a switch. The three treatments, and combinations thereof, are indicated by the illumination of three 6-W lights with a red, green, and white frosted jewel, respectively, in the stimulus light panel mounted on the primate restraining chair. No ilumination of the lights indicates a rest period, i.e., no treatments, and is programmed by having the programmer "on" with no treatment scheduled. 